\title{
Visfatin Increases VEGF-Dependent Angiogenesis of Endothelial Progenitor Cells during Osteoarthritis Progression
}

\author{
Chun-Hao Tsai ${ }^{1,2}{ }^{\oplus}$, Shan-Chi Liu ${ }^{3}$, Wen-Hui Chung ${ }^{4}$, Shih-Wei Wang ${ }^{5,6}{ }^{(\mathbb{D}}$, Min-Huan Wu ${ }^{7,8, *}$ \\ and Chih-Hsin Tang 4,9,10,*(D) \\ 1 Department of Sports Medicine, College of Health Care, China Medical University, Taichung 404, Taiwan; \\ ritsai8615@gmail.com \\ 2 Department of Orthopedic Surgery, China Medical University Hospital, Taichung 404, Taiwan \\ 3 Department of Medical Education and Research, China Medical University Beigang Hospital, Yunlin 651, \\ Taiwan; sdsaw.tw@yahoo.com.tw \\ 4 Department of Pharmacology, School of Medicine, China Medical University, Taichung 404, Taiwan; \\ sandy780717@gmail.com \\ 5 Department of Medicine, Mackay Medical College, New Taipei City 252, Taiwan; shihwei@mmc.edu.tw \\ 6 Graduate Institute of Natural Products, College of Pharmacy, Kaohsiung Medical University, \\ Kaohsiung 807, Taiwan \\ 7 Physical Education Office, Tunghai University, Taichung 407, Taiwan \\ 8 Sports Recreation and Health Management Continuing Studies, Tunghai University, Taichung 807, Taiwan \\ 9 Chinese Medicine Research Center, China Medical University, Taichung 404, Taiwan \\ 10 Department of Biotechnology, College of Health Science, Asia University, Taichung 41354, Taiwan \\ * Correspondence: mhwu@thu.edu.tw (M.-H.W.); chtang@mail.cmu.edu.tw (C.-H.T.)
}

Received: 8 April 2020; Accepted: 22 May 2020; Published: 25 May 2020

\begin{abstract}
Osteoarthritis (OA) pannus contains a network of neovascularization that is formed and maintained by angiogenesis, which is promoted by vascular endothelial growth factor (VEGF). Bone marrow-derived endothelial progenitor cells (EPCs) are involved in VEGF-induced vessel formation in OA. The adipokine visfatin stimulates the release of inflammatory cytokines during OA progression. In this study, we found significantly higher visfatin and VEGF serum concentrations in patients with OA compared with healthy controls. We describe how visfatin enhanced VEGF expression in human OA synovial fibroblasts (OASFs) and facilitated EPC migration and tube formation. Treatment of OASFs with PI3K and Akt inhibitors or siRNAs attenuated the effects of visfatin on VEGF synthesis and EPC angiogenesis. We also describe how miR-485-5p negatively regulated visfatin-induced promotion of VEGF expression and EPC angiogenesis. In our OA rat model, visfatin shRNA was capable of inhibiting visfatin and rescuing EPC angiogenesis and pathologic changes. We detail how visfatin affected VEGF expression and EPC angiogenesis in OASFs by inhibiting miR-485-5p synthesis through the PI3K and Akt signaling pathways.
\end{abstract}

Keywords: osteoarthritis; visfatin; VEGF; miR-485-5p; angiogenesis

\section{Introduction}

Osteoarthritis (OA) is a common age-related and chronic degenerative joint disorder that affects all body joints. Major symptoms of OA include cartilage degradation, osteophyte formation, bone remodeling, neoangiogenesis, and synovial inflammation [1], which are associated with pain, physical disability, and substantial impairments in quality of life. The synovium plays an important role in the pathogenesis of OA. The synthesis of chondrolytic enzymes and proinflammatory mediators by the 
inflamed synovium leads to cartilage destruction, which in turn enhances synovial inflammation [2,3]. OA synovial fibroblasts (OASFs) sustain arthritic pathology by excreting chondrolytic enzymes and inflammatory mediators $[2,4,5]$. In theory, synovium-targeted therapy could slow OA progression and mitigate the disease symptoms [6,7].

OA pannus contains a network of neovascularization that is formed and maintained by angiogenesis, which is promoted by vascular endothelial growth factor (VEGF) [8]. New vessel formation also involves endothelial progenitor cells (EPCs) $[9,10]$, which contain the cell surface markers CD133, CD34, and vascular endothelial growth factor receptor-2 (VEGFR2), all of which facilitate postnatal vasculogenesis [11] and exert regenerative effects [12]. VEGF is well recognized for its ability to increase EPC proliferation and migration, besides facilitating angiogenesis [12]. Importantly, VEGF-induced promotion of EPCs facilitates angiogenesis and the development of OA $[13,14]$. Thus, EPC-dependent angiogenesis shows promise as a new target for OA treatment.

Obesity reportedly increases the risk of developing OA [15,16], although the underlying mechanisms for this risk association are unknown. What is known is that adipokines, multifunctional molecules secreted by the adipose tissue, act as an intersecting link between obesity and OA by modulating the activities of cartilage, synovium, bone, and various immune cells $[15,17]$. Visfatin is a proinflammatory adipokine produced by visceral white adipose tissue and is found in the bone marrow, skeletal muscles, and liver [18]. Upregulated serum levels of visfatin are found in OA patients $[19,20]$. Visfatin also plays a role in damage of the synovial joint [16]. The present study aims to investigate the connection between visfatin and EPC-dependent angiogenesis during OA progression. The evidence indicates that visfatin is an appropriate therapeutic target in OA.

\section{Materials and Methods}

\subsection{Materials}

Cell culture supplements were bought from Invitrogen (Carlsbad, CA, USA). Antibodies against p85, Akt, p-p85, p-Akt, VEGF, and $\beta$-actin were all bought from Santa Cruz (Santa Cruz, CA, USA). All ON-TARGETplus siRNAs (pool form) were bought from Dharmacon (Lafayette, CO, USA). The miR-485-5p mimic and mimic control were obtained from Thermo Fisher (Waltham, MA, USA). qPCR primers and probes, as well as Taqman ${ }^{\circledR}$ one-step PCR Master Mix, were supplied by Applied Biosystems (Foster City, CA, USA). Ly294002 and the Akt inhibitor (124005) were purchased from Sigma-Aldrich (St. Louis, MO, USA).

\subsection{Cell Culture}

Synovial tissue from the suprapatellar pouch of the knee was obtained from patients diagnosed with Ahlbäck stage IV OA. Synovial fibroblasts were cultured in DMEM medium supplemented with $10 \%$ fetal bovine serum (FBS), penicillin (50 units $/ \mathrm{mL})$, and streptomycin $(50 \mu \mathrm{g} / \mathrm{mL})$, as previous reports described [21,22].

Human primary EPCs were prepared according to our previously described protocol [23,24]. Cells were maintained in a humidified incubator at $37^{\circ} \mathrm{C}, 5 \% \mathrm{CO}_{2}$.

\subsection{Clinical Samples}

Blood samples were obtained from patients with primary OA (we excluded any cases with underlying immuno-inflammatory rheumatic diseases or crystal deposition arthritis) of the knee undergoing knee replacement surgery and also from those undergoing arthroscopy after trauma/mechanical patellofemoral syndrome (who served as healthy controls) in China Medical University Hospital, Taichung, Taiwan. The study protocol was approved by the Institutional Review Board (IRB) of China Medical University Hospital, and all methods were performed in accordance with the IRB's guidelines and regulations. Informed written consent was obtained from all patients. 


\subsection{RT-qPCR of $m R N A$ and miRNA}

Total RNA was extracted from OASFs by TRIzol; reverse transcription used $1 \mu \mathrm{g}$ of total RNA transcribed into cDNA by oligo (dT) primers. RT-qPCR used the Taqman ${ }^{\circledR}$ One-Step RT-PCR Master $\operatorname{Mix}[25,26]$.

\subsection{Western Blot Analysis}

Cell lysates were separated by SDS-PAGE electrophoresis then transferred to polyvinylidene difluoride membranes, following the method described in our previous work $[27,28]$. After blocking, the membranes were incubated with primary antibodies and then secondary antibodies. Enhanced chemiluminescence of the blots was visualized with the UVP Biospectrum system (UVP, Upland, CA, USA) [29-31].

\subsection{ELISA Assay}

OASFs were cultured and stimulated with visfatin for $24 \mathrm{~h}$ with or without the transfection of siRNAs or the treatment with inhibitors. The conditioned medium (CM) was collected, and VEGF levels were quantified by a VEGF-A ELISA kit (Peprotech, Rocky Hill, NJ, USA).

\subsection{Transient Transfection}

Human synovial fibroblasts were cultured in a 6-well plate, and miR-150-5p mimic or visfatinshRNA was transfected into the cells by Lipofectamine ${ }^{\mathrm{TM}} 2000$.

ON-TARGETplus siRNAs (100 nM) was transiently transfected with DharmaFECT1 transfection reagent, according to the manufacturer's instructions.

\subsection{Plasmid Construction and Luciferase Assays}

Wild-type and mutant VEGF 3'-UTR plasmids were obtained from Invitrogen (Carlsbad, CA, USA). Luciferase activity was examined using the method described in our previous reports $[2,21,32]$.

\subsection{EPC Migration and Tube Formation Assays}

EPCs were treated with OASF CM for $24 \mathrm{~h}$. EPC migration and tube formation were examined using the methods described in our previous study [33].

\subsection{In Vivo Matrigel Plug Assay}

Four-week-old male nude mice were subcutaneously injected with $0.15 \mathrm{~mL}$ of Matrigel containing the indicated OASF CM. On day 7, the Matrigel plugs were harvested, and hemoglobin concentrations were measured according to previously described procedures $[14,34,35]$.

\subsection{Experimental OA Model}

Sprague-Dawley (SD) rats (8 weeks of age, weighing 300-350 g) were procured from the National Laboratory Animal Center in Taiwan and maintained under conditions complying with the Guidelines of the Animal Care Committee of China Medical University, Taichung, Taiwan. We followed an established protocol for our anterior cruciate ligament transection (ACLT) rat model to induce OA [36]. In brief, the left knee was prepared in a surgically sterile fashion. The ACL fibers were transected with a scalpel, and the entire medial meniscus was excised via the medial parapatellar mini-arthrotomy approach. The joint surface was washed with sterile saline solution, and both capsule and skin were sutured after ACL transection and medial meniscectomy. The left knee joint served as the sham-operated control. After surgery (day 0), the rats were divided into 3 groups: a control group, a control shRNA-transfected ACLT group, and a visfatin shRNA-transfected ACLT group. For 6 weeks, the control shRNA-transfected ACLT group and visfatin shRNA-transfected ACLT group were given 
weekly intra-articular injections of $\sim 7.1 \times 10^{6}$ plaque-forming units (PFU) of control and visfatin shRNA. All rats were allowed to move freely in plastic cages until necropsy at 10 weeks post-surgery.

\subsection{Micro-Computed Tomography (Micro-CT) Imaging}

The micro-computed tomography (micro-CT) assessment protocol was based upon our previous publications [14,35]. Rat knee joints were extracted promptly after sacrifice and fixed in 3.7\% formaldehyde for micro-CT imaging. Three-dimensional microstructural volumes from micro-CT scans were analyzed by Skyscan software (CTAn; Bruker) [14].

\subsection{Statistics}

All statistical analyses were carried out using GraphPad Prism 5.0 (GraphPad Software), and all values are expressed as mean \pm S.D. Differences between selected pairs from the experimental groups were analyzed for statistical significance using the paired sample $t$-test for in vitro analyses and one-way ANOVA followed by Bonferroni testing for in vivo analyses. Correlations between plasma VEGF and visfatin were calculated using Spearman's rank correlation coefficient (R). The statistical difference was considered to be significant if the $p$-value was $<0.05$.

\section{Results}

\subsection{A Positive Correlation Exists between Visfatin and VEGF Expression in $O A$}

In our initial exploration of visfatin and VEGF expression in OA development, ELISA test results revealed significantly higher visfatin and VEGF serum concentrations in patients with OA compared with healthy controls (Figure 1A,B and Supplementary Material Tables S1 and S2). Serum visfatin and VEGF concentrations were positively correlated (Figure 1C).

(A)
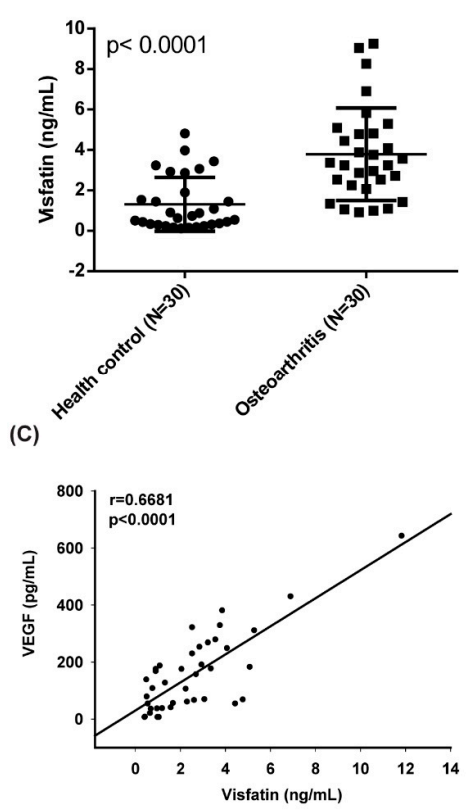

(B)

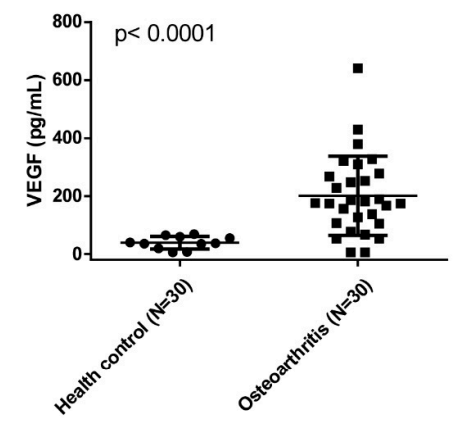

Figure 1. Visfatin expression is positively correlated with vascular endothelial growth factor (VEGF) expression in OA patients. (A,B) ELISA analysis showing higher serum visfatin and VEGF levels in osteoarthritis (OA) patients $(n=30)$ compared with healthy controls $(n=30)$. Mann-Whitney testing was applied in Figure 1A,B. (C) Correlation between levels of visfatin and VEGF expression in serum samples retrieved from $O A$ patients. 


\subsection{Visfatin Increases VEGF Expression and EPC Angiogenesis in Human OASFs}

No detailed information exists regarding any crosstalk between visfatin and VEGF in the pathogenesis of OA or on how such an interaction may influence EPC angiogenesis. Here, we found that visfatin (1-30 $\mathrm{ng} / \mathrm{mL}$ ) dose-dependently stimulated transcription of VEGF mRNA and VEGF translation at the protein level (Figure 2A,B) as well as the excretion of the VEGF protein by OASFs (Figure 2C).

As the formation of new blood vessels depends on the migration of EPCs through the capillary basement membrane [37], we analyzed the role of visfatin in EPC migratory activity. The Transwell assay revealed a dramatic increase in EPC migration after their incubation with CM from visfatin-treated OASFs, while the tube formation assay showed that visfatin-treated OASFs dose-dependently facilitated the formation and reorganization of capillary-like network structures (Figure 2D,E; Supplementary Material Figure S1).

(A)
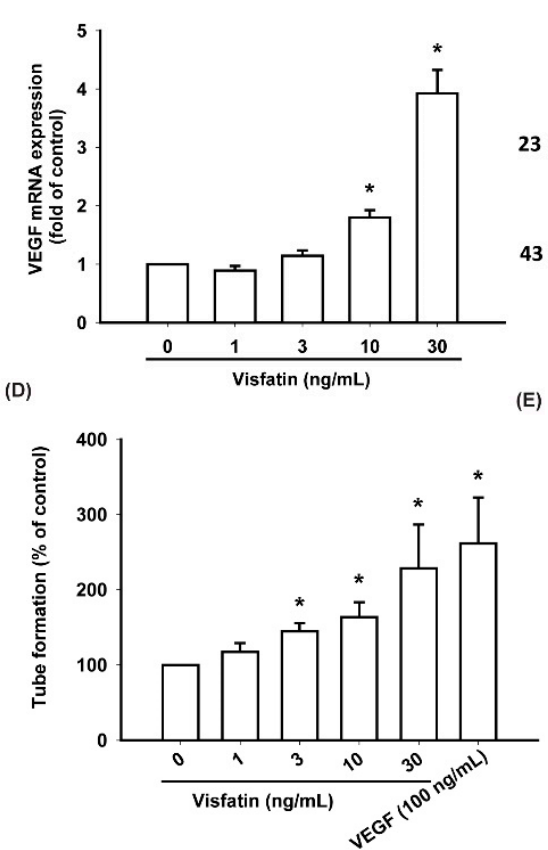

(B)
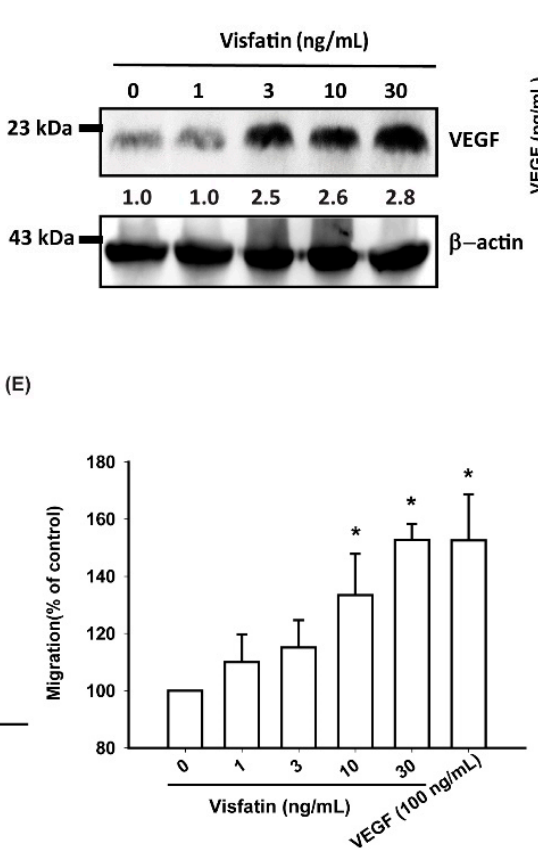

(C)

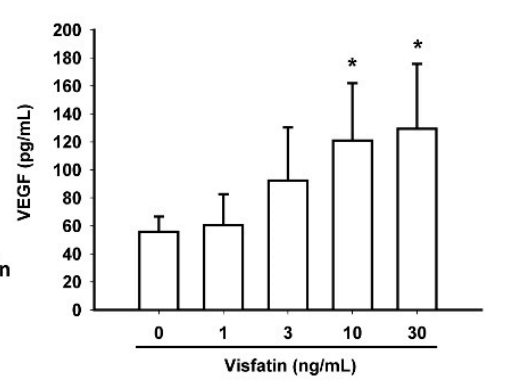

Figure 2. Visfatin stimulates VEGF expression and endothelial progenitor cells (EPC) angiogenesis in OA synovial fibroblasts (OASFs). (A-C) OASFs were incubated with visfatin (1-30 ng/mL) for $24 \mathrm{~h}$, and VEGF expression was examined by RT-qPCR, Western blot, and ELISA analysis. (D,E) The conditioned medium $(\mathrm{CM})$ was then collected and applied to EPCs. EPC tube formation and migration were measured; ${ }^{*} p<0.05$ compared with the control group.

\subsection{Visfatin Promotes VEGF Production and EPC Angiogenesis via the PI3K and Akt Signaling Pathways}

The PI3K signaling pathway modulates several cellular functions, including angiogenesis $[33,38]$. We explored the role of PI3K in visfatin-enhanced VEGF expression by pretreating OASFs with a PI3K inhibitor (Ly294002) or transfecting them with p85 siRNA. Quantitative reverse transcription PCR (RT-qPCR), ELISA, and Western blot assays confirmed that the PI3K inhibitor and p85 siRNA not only significantly reduced visfatin-increased VEGF expression in OASFs (Figure 3A-C) but also inhibited visfatin-promoted EPC migration and tube formation (Figure 3D,E). Western blot analysis demonstrated that visfatin time-dependently promoted p 85 phosphorylation (Figure 3F). Transfection of cells with p85 siRNA reduced p85 expression and phosphorylation (Figure 3G).

Akt is a common downstream signaling molecule of PI3K and a mediator of EPC angiogenesis $[39,40]$. When we treated OASFs with an Akt inhibitor or transfected them with 
Akt siRNA prior to visfatin administration, we observed marked reductions in visfatin-induced increases in VEGF expression, EPC migration, and tube formation (Figure 4A-E). In Western blot analysis, visfatin time-dependently promoted Akt phosphorylation (Figure 4F), which was inhibited by the treatment with a PI3K inhibitor (Figure 4G). Transfection of cells with Akt siRNA reduced Akt expression and phosphorylation (Figure $4 \mathrm{H}$ ).

(A)

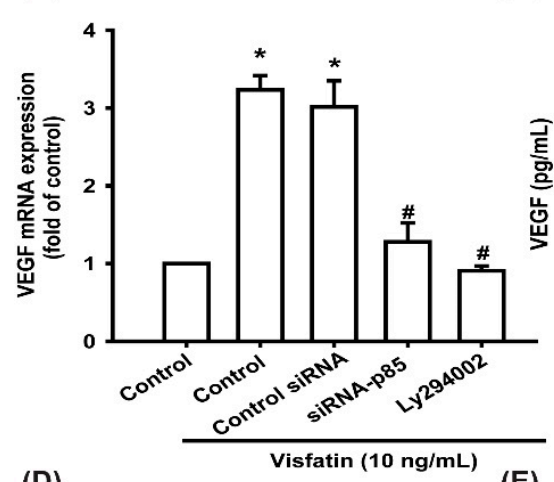

(D)

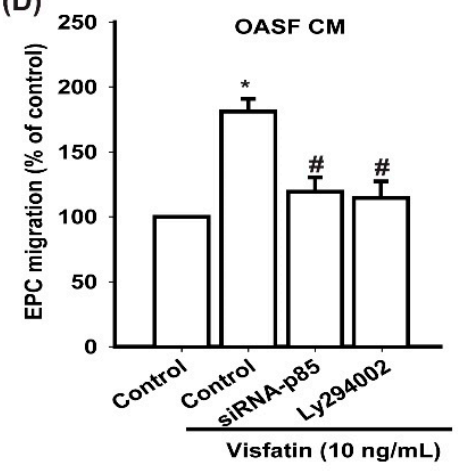

(B)

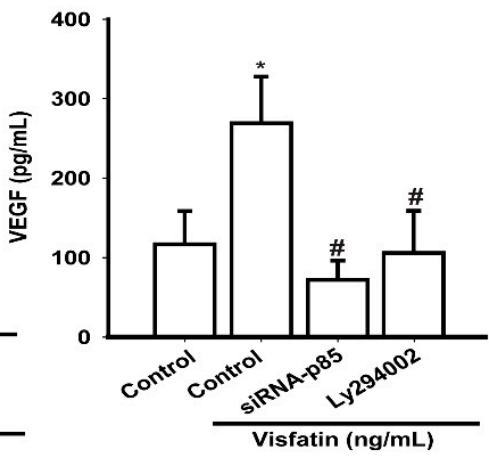

(E)
(C)

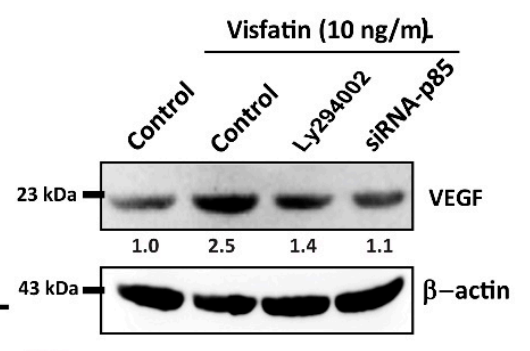

(F)

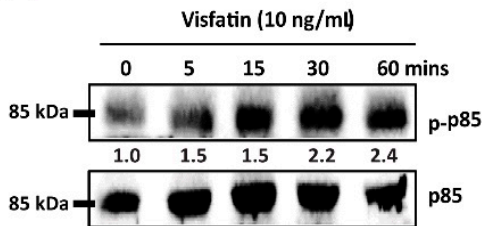

(G)

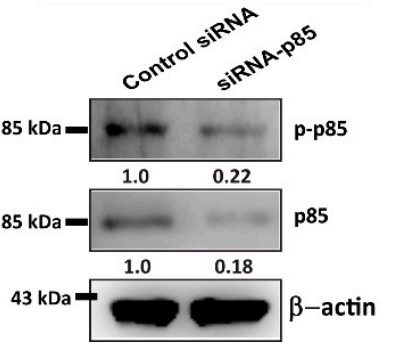

Figure 3. The PI3K pathway is involved in visfatin-induced VEGF synthesis and EPC angiogenesis. (A-C) OASFs were pretreated with a PI3K inhibitor (Ly294002) or transfected with p85 siRNA, then incubated with visfatin for $24 \mathrm{~h}$. VEGF levels were examined by RT-qPCR, ELISA, and Western blot. (D,E) The CM was then collected and applied to EPCs. EPC tube formation and migration were measured. (F) OASFs were incubated with visfatin for the indicated time intervals, and p85 phosphorylation was examined by Western blot. (G) OASFs were transfected with p85 siRNA, and p85 and p-p85 expression was examined by Western blot; ${ }^{*} p<0.05$ compared with the control group; ${ }^{\#} p<0.05$ compared with the visfatin-treated group. 


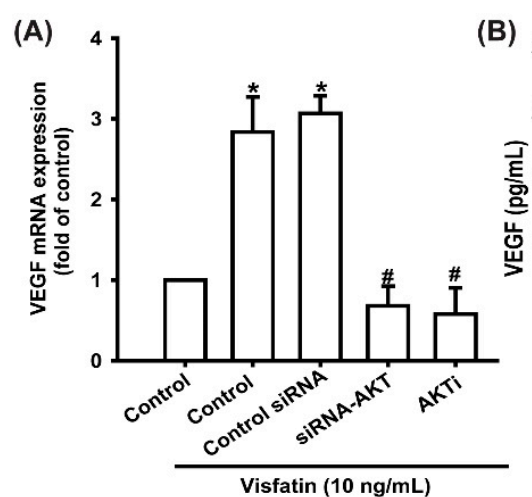

(D)

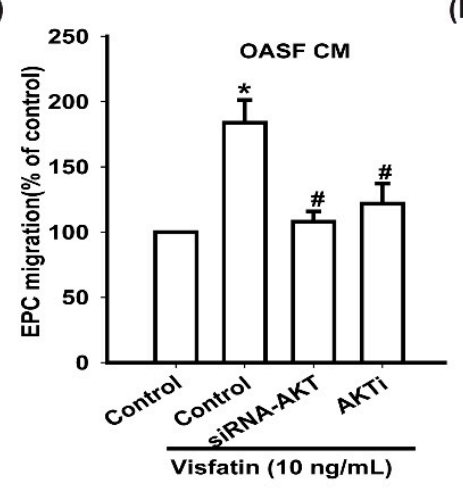

(H)

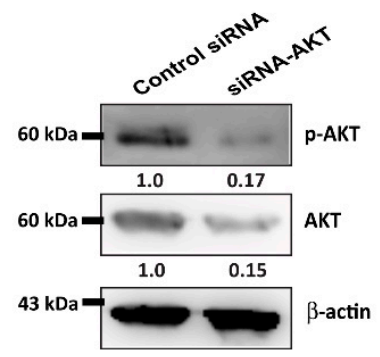

(C)

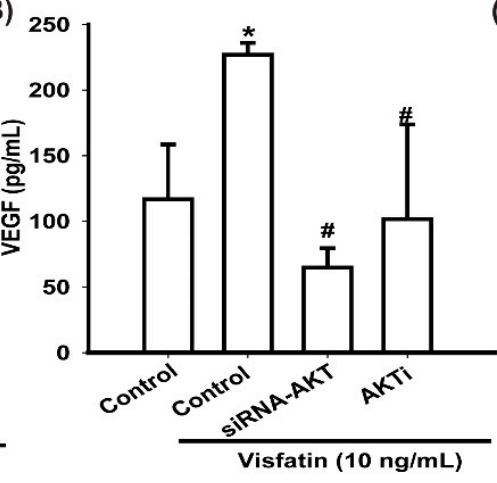

(E)

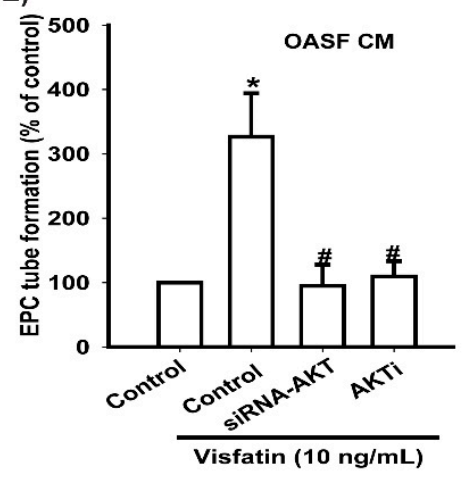

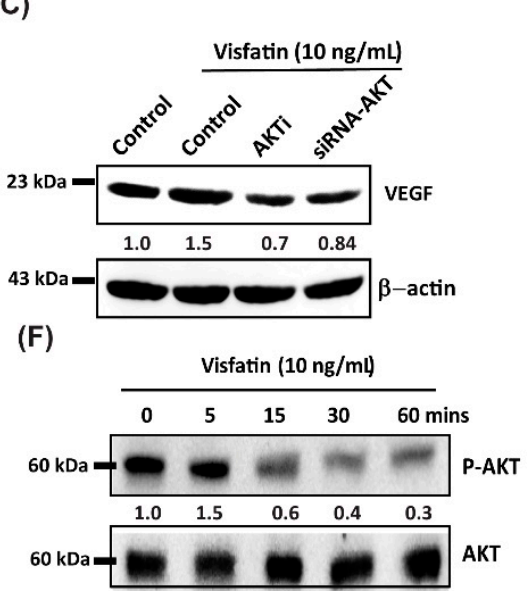

(G)

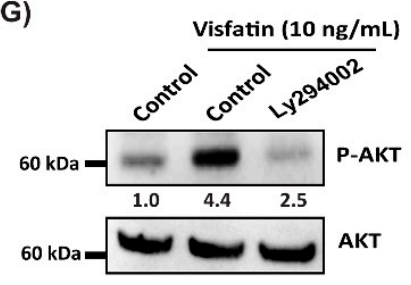

Figure 4. The Akt pathway is involved in visfatin-induced VEGF synthesis and EPC angiogenesis. (A-C) OASFs were pretreated with an Akt inhibitor or transfected with Akt siRNA, then incubated with visfatin for $24 \mathrm{~h}$. VEGF levels were examined by RT-qPCR, ELISA and Western blot. (D,E) The CM was then collected and applied to EPCs. EPC tube formation and migration were measured. (F,G) OASFs were incubated with visfatin for the indicated time intervals or pretreated with a PI3K inhibitor and then stimulated with visfatin, and Akt phosphorylation was examined by Western blot. (H) OASFs were transfected with Akt siRNA, then Akt and p-Akt expression was examined by Western blot; ${ }^{*} p<0.05$ compared with the control group; ${ }^{*} p<0.05$ compared with the visfatin-treated group.

\subsection{Visfatin Increases VEGF Production and EPC Angiogenesis via the Inhibition of miR-485-5p Synthesis}

miRNA expression patterns differ between OA and normal cartilage, and several miRNAs are implicated in OA pathogenesis [22,41]. Using open-source software (TargetScan, miRMap, RNAhybrid, and miRWalk), we sought to identify miRNAs that could potentially interfere with VEGF transcription. Of five candidate miRNAs that we found could bind to the 3' untranslated region (UTR) of VEGF mRNA, miR-485-5p expression was reduced to the greatest extent after visfatin administration (Figure 5A; Supplementary Material Figure S2). Stimulating OASFs with visfatin concentration-dependently inhibited miR-485-5p expression (Figure 5B). To further determine whether visfatin stimulates VEGF expression and EPC angiogenesis by inhibiting miR-485-5p expression, we transfected OASFs with an miR-485-5p mimic and observed reductions in visfatin-enhanced VEGF expression, EPC migration, and tube formation (Figure $5 \mathrm{C}-\mathrm{G}$ ). 
We then sought to determine whether visfatin promotes EPC angiogenesis by inhibiting miR-485-5p in vivo. The Matrigel plug assay showed that CM from visfatin-treated OASFs increased vessel formation in vivo (Figure 6A), while the miR-485-5p mimic abolished visfatin-induced promotion of vessel formation (Figure 6A). Immunohistochemistry (IHC) staining indicated that the miR-485-5p mimic antagonized visfatin-facilitated increases in the expression of the vessel marker CD31 and of the levels of VEGF and the EPC-specific markers CD34 and CD133 (Figure 6B).

To determine whether miR-485-3p controls transcription of the VEGF gene, we examined the effects of a luciferase reporter vector containing the wild-type $3^{\prime}$ UTR of VEGF mRNA (wt-VEGF-3'UTR) and a mutated vector harboring mismatches in the predicted miR-485-3p binding site (mt-VEGF-3'UTR) (Figure 6C). The miR-485-5p mimic inhibited visfatin-increased luciferase activity in the wt-VEGF-3'UTR plasmid but not in the mt-VEGF-3'UTR plasmid (Figure 6C), indicating that miR-485-5p suppresses VEGF transcription through binding to the 3'UTR region of human VEGF mRNA. In addition, the PI3K and Akt inhibitors markedly reversed visfatin-reduced miR-485-5p expression (Figure 6D). Transfection of cells with the miR-485-5p mimic increased miR-485-5p expression (Figure 6E).

(A)

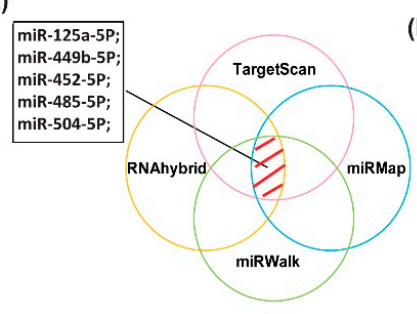

(D)

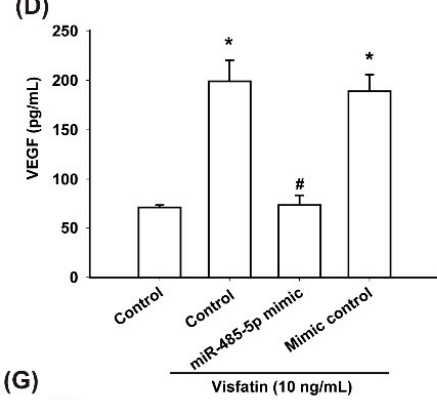

(G)

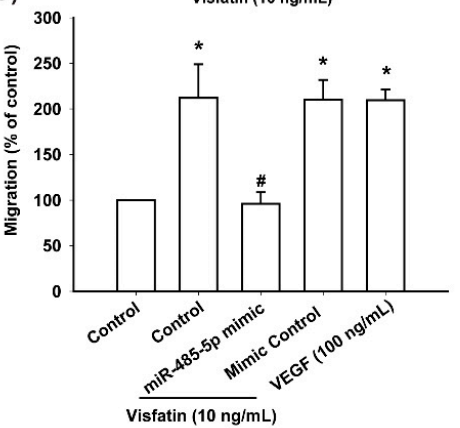

(B)

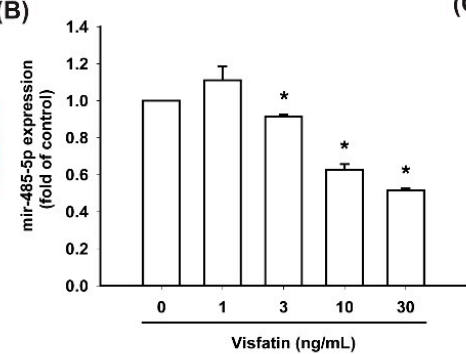

(E)

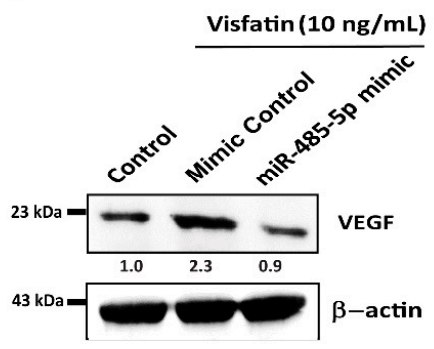

(C)

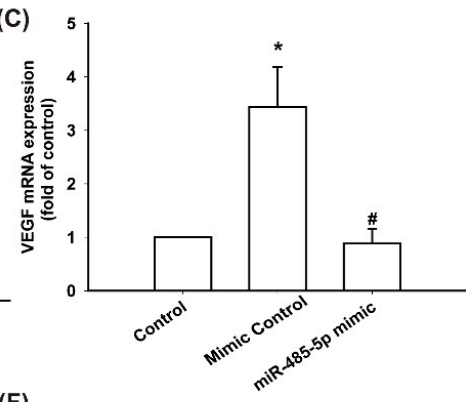

(F)

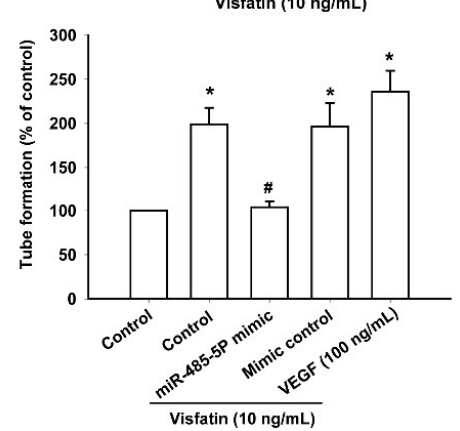

Figure 5. Visfatin promotes VEGF production and EPC angiogenesis by suppressing miR-485-5p. (A) Open-source software (TargetScan, miRMap, RNAhybrid, and miRWalk) were used to identify which miRNAs could possibly interfere with VEGF transcription. (B) OASFs were incubated with visfatin (1-30 ng/mL). miR-485-5p expression was examined by RT-qPCR. (C-E) OASFs were transfected with the miR-485-5p mimic and then stimulated with visfatin. VEGF levels were examined by RT-qPCR, ELISA, and Western blot. (F,G) The CM was then collected and applied to EPCs. EPC tube formation and migration was measured; ${ }^{*} p<0.05$ compared with the control group; ${ }^{\#} p<0.05$ compared with the visfatin-treated group. 
(A)

(B)

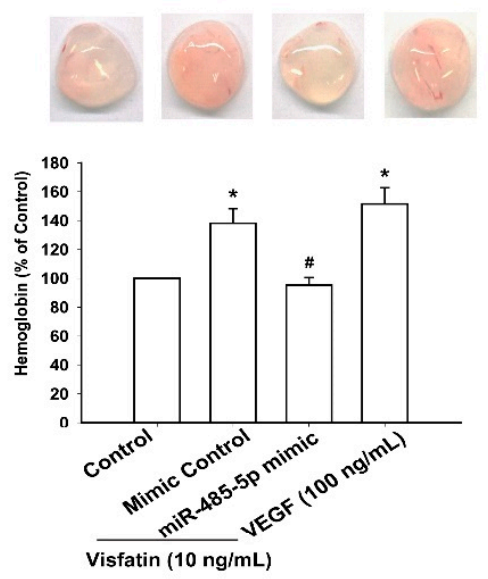

(C)

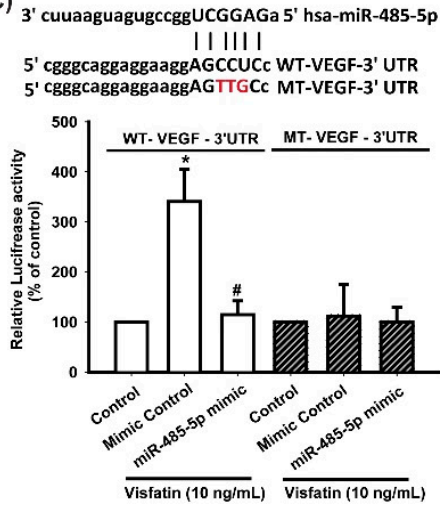

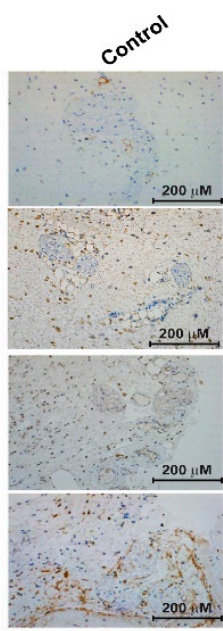

(D)

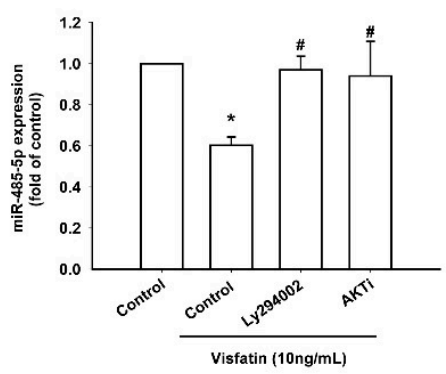

Visfatin $(10 \mathrm{ng} / \mathrm{mL})$

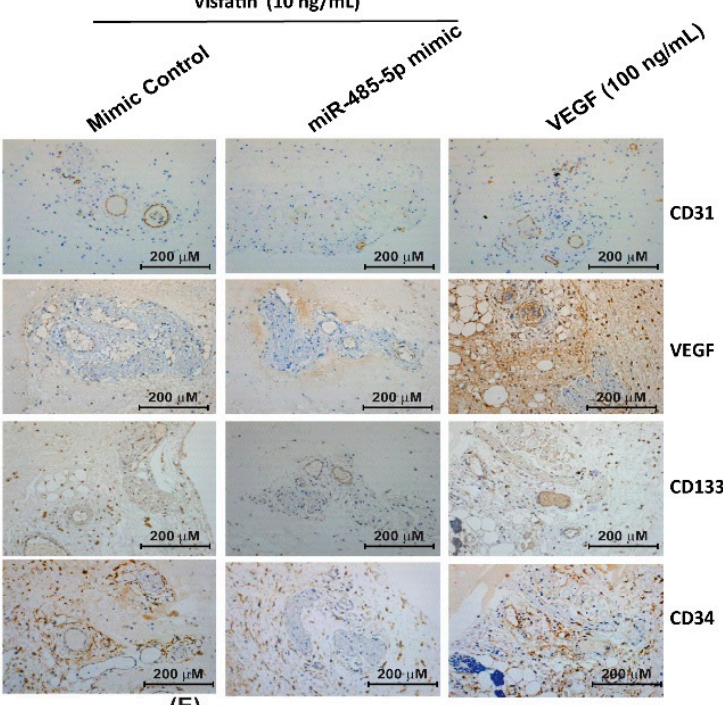

(E)

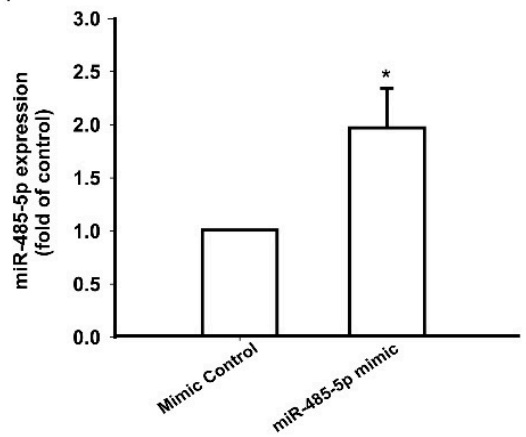

Figure 6. Visfatin suppresses miR-485-5p synthesis via the PI3K and Akt pathways. (A) Matrigel plugs containing OASF CM were subcutaneously injected into the flanks of nude mice. After 7 days, the plugs were photographed, and hemoglobin levels were quantified. (B) Specimens from the plugs were immunostained with antibodies against CD31, VEGF, CD34, and CD133. (C) Schematic 3'UTR representation of human VEGF containing the miR-485-5p binding site. (D) OASFs were transfected with the indicated luciferase plasmid with or without the miR-485-5p mimic, then stimulated with visfatin. Relative luciferase activity was examined. (E) OASFs were transfected with the miR-485-5p mimic, and miR-485-5p expression was examined by qPCR; ${ }^{*} p<0.05$ compared with the control group; \# $p<0.05$ compared with the visfatin-treated group.

\subsection{Visfatin Knockdown Mitigates EPC Angiogenesis and OA Severity In Vivo}

The transfection of human OASFs with visfatin shRNA reduced visfatin and VEGF expression (Figure 7A), EPC migration and tube formation (Figure 7B,C), and angiogenesis in vivo (Figure 7D,E).

We then investigated the effects of shRNA-mediated visfatin knockdown on OA severity in our ACLT model. Micro-CT imaging revealed that visfatin shRNA restored the integrity of subchondral bone architecture in ACLT rats (Figure 7F). In comparison to the control samples, subchondral bone from rats with ACLT-induced OA that underwent control shRNA transfection had significantly lower bone volume (BV), BMD (bone mineral density), bone surface (BS), and trabecular (Tb) number and thickness (Th) and higher Tb spacing (Figure 7G). All of these ACLT-induced effects were reversed by visfatin shRNA transfection. IHC and Safranin-O staining demonstrated lower cartilage thickness and significantly higher expression of VEGF in ACLT samples. ACLT-induced histologic changes were reversed by visfatin shRNA transfection (Figure $7 \mathrm{H}$ ). 


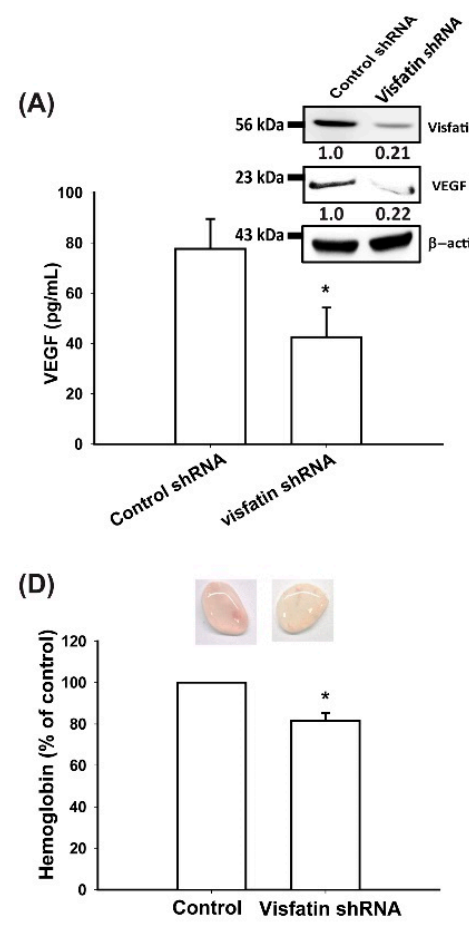

(B)

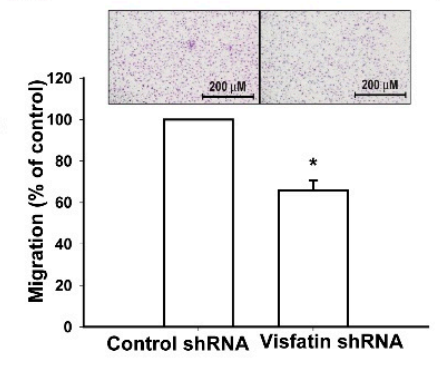

(E)

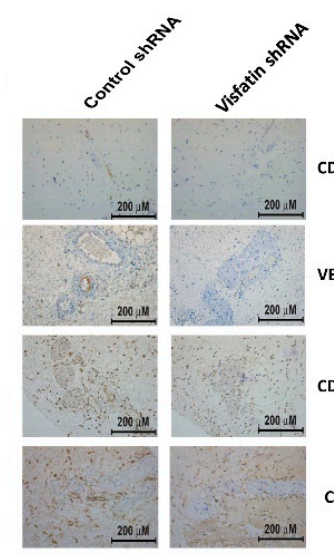

(F)

(H)

(C)
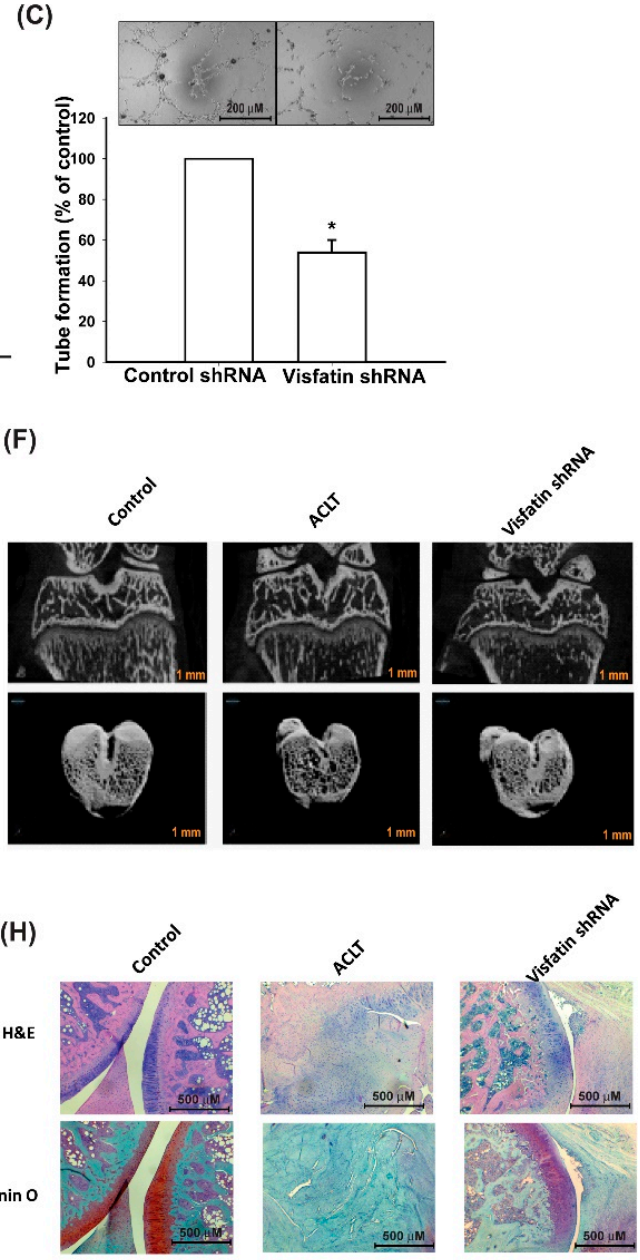

(G)
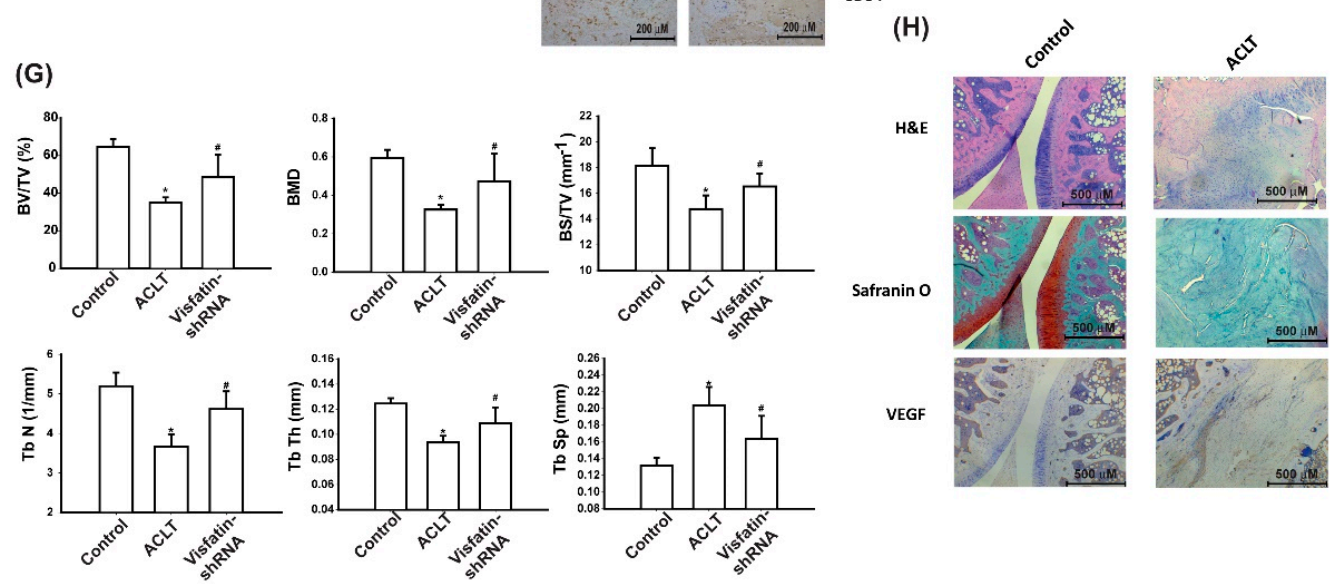

VEGF
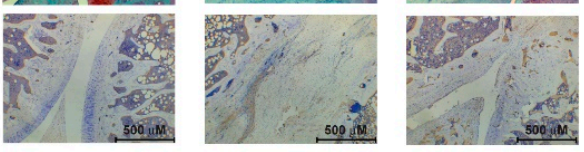

Figure 7. Visfatin knockdown reduces EPC angiogenesis and pathological severity of OA. (A) OASFs were transfected with visfatin shRNA. Visfatin and VEGF expression was examined by Western blot and ELISA. (B,C) The CM was then collected and applied to EPCs. EPC tube formation and migration were measured. (D) Matrigel plugs containing OASF CM were subcutaneously injected into the flanks of nude mice. After 7 days, the plugs were photographed, and hemoglobin levels were quantified. (E) Specimens from the plugs were immunostained with antibodies against CD31, VEGF, CD34, and CD133. (F) Micro-CT images of the control knee, ACLT knee, and visfatin shRNA-transfected ACLT knee. (G) Micro-computed tomography (CT) parameters, including bone volume (BV), bone mineral density (BMD), bone surface (BS), trabecular (Tb) number and thickness (Th), and space. (H) Specimens from the knee were immunostained with Safranin-O, and anti-VEGF antibodies; ${ }^{*} p<0.05$ compared with the control group; ${ }^{\#} p<0.05$ compared with the visfatin-treated group.

\section{Discussion}

OA is the most common form of arthritis and a primary cause of disability [1]. Although much is unclear as to the pathogenesis of $\mathrm{OA}$, it is known that synovium inflammation plays a pivotal role [42], so synovium-targeted therapy could theoretically slow OA progression and lessen the severity of symptoms $[6,43]$. Pannus formation and neovascularization also play important roles in OA 
development [8]. VEGF-induced stimulation of angiogenesis is a critical step during OA progression $[8,44]$. We have previously reported that visfatin promotes the production of the proinflammatory cytokines IL-6 and TNF- $\alpha$ in OASFs [16]. However, the effects of visfatin on VEGF expression and EPC angiogenesis are not clear. In this study, we found higher levels of visfatin and VEGF in patients with OA compared with healthy controls. We also found that visfatin stimulates EPC angiogenesis in OASFs by increasing VEGF expression after inhibiting miR-485-5p synthesis through the PI3K and Akt signaling pathways.

Previous research has demonstrated the upregulated visfatin concentrations in synovial fluid from OA patients compared with that from healthy individuals $[19,20]$. In this study, we confirmed that visfatin and VEGF levels are higher in serum from OA patients than in that from healthy controls. Our investigation revealed positive correlations between visfatin and VEGF concentrations. According to the evidence presented, VEGF may serve as a target molecule for the visfatin signaling pathway, which facilitates EPC angiogenesis in human OASFs. Knockdown of visfatin inhibited ACLT-induced OA and the expression of EPC and vessel markers in vivo. Visfatin is clearly a critical molecular target in OA therapy. To the best of our knowledge, no investigations have identified any specific visfatin receptor. Thus, further explorations are needed to determine which receptors mediate visfatin-induced VEGF expression and EPC angiogenesis.

The activation of the PI3K signaling pathway is essential for regulating multiple cellular functions [45], including angiogenesis and metastasis [33,46]. Our evidence shows that visfatin facilitates p85 phosphorylation, while a PI3K inhibitor and siRNA treatment diminished visfatin-enhanced VEGF production. These compounds effectively attenuated visfatin-induced promotion of EPC migration and tube formation. PI3K-dependent Akt activation is critical for controlling angiogenesis [47,48]. We found that an Akt inhibitor and siRNA treatment reversed visfatin-induced increases in VEGF expression and EPC migration and tube formation, indicating that Akt is required for visfatin-promoted VEGF production and EPC angiogenesis. Our findings also reveal that visfatin facilitates Akt phosphorylation. PI3K inhibitor treatment reduced visfatin-induced Akt phosphorylation, suggesting that PI3K-dependent Akt activation regulates visfatin-mediated VEGF expression and EPC angiogenesis in human OASFs. However, we did not examine which upstream molecules of PI3K were affected by visfatin stimulation. How visfatin activates PI3K/Akt signaling requires further examination.

It has been stated that miRNAs effectively post-transcriptionally regulate gene expression [49]. In OA, several miRNAs show aberrant expression levels and are capable of regulating the expression of inflammatory pathways [49]. It is speculated that pharmacotherapy capable of regulating miRNA expression would reduce the inflammatory process in OA and assist with the management of this disease $[49,50]$. We searched open-source miRNA software to determine whether miR-485-5p interferes with VEGF transcription. We found that visfatin stimulation reduces miR-485-5p synthesis and that transfecting OASFs with an miR-485-5p mimic mitigates visfatin-stimulated VEGF expression and EPC angiogenesis. PI3K and Akt inhibitor treatments rescued visfatin-induced inhibition of miR-485-5p expression, indicating that visfatin facilitates VEGF production and EPC angiogenesis by reducing miR-485-5p expression via the PI3K and Akt signaling cascades.

Histopathological features of OA that are illustrated by the ACLT model include articular cartilage destruction and infiltration of inflammatory cells [36]. In this study, micro-CT imaging and IHC staining of the ACLT joints revealed articular cartilage erosion, which was attenuated by visfatin shRNA. IHC staining also revealed that visfatin shRNA reduced the levels of a vessel marker (VEGF), suggesting that the effect of visfatin in ACLT rats might be due to the expression of angiogenic mediators.

In order to ensure the confidentiality and anonymity of our study participants, we did not record demographic details of age, gender, body mass index, or any other general information. Lacking these details means that we were unable to compare demographic data with visfatin and VEGF expression. 


\section{Conclusions}

Our study shows that visfatin increases VEGF expression and promotes EPC angiogenesis in OASFs by inhibiting miR-485-5p synthesis via PI3K and Akt signaling. Thus, visfatin is an appropriate therapeutic target in OA. Our results enrich our knowledge about the involvement of OASFs in OA and may lead to more effective therapies. Further studies are needed to explore which antibodies or small molecules against visfatin could be used to treat OA.

Supplementary Materials: The following are available online at http://www.mdpi.com/2073-4409/9/5/1315/s1, Figure S1: The image of figure 2D and 2E, Figure S2. The miRNAs expression after Visfatin treatment in human OASFs, Table S1: Visfatin levels in serum (Mann-Whitney test), Table S2: VEGF levels in serum (Mann-Whitney test).

Author Contributions: M.-H.W. and C.-H.T. (Chih-Hsin Tang) initiated the research project. C.-H.T. (Chun-Hao Tsai), S.-C.L., and W.-H.C. performed the research. C.-H.T. (Chun-Hao Tsai) and S.-W.W. provided the material. M.-H.W. and C.-H.T. (Chih-Hsin Tang) wrote the paper. All authors have read and agreed to the published version of the manuscript.

Funding: This study was supported by grants from Taiwan's Ministry of Science and Technology (MOST 108-2320-B-039-026-; MOST 108-2320-B-039-064); China Medical University (CMU 108-MF-66) and China Medical University Hospital (DMR-109-159).

Acknowledgments: We would like to thank Iona J. MacDonald for her English language revision of this manuscript.

Conflicts of Interest: There are no conflicts of interest to disclose.

\section{References}

1. Yuan, X.; Meng, H.; Wang, Y.; Peng, J.; Guo, Q.; Wang, A.; Lu, S. Bone-cartilage interface crosstalk in osteoarthritis: Potential pathways and future therapeutic strategies. Osteoarthr. Cartil. 2014, 22, 1077-1089. [CrossRef] [PubMed]

2. Kuo, S.J.; Yang, W.H.; Liu, S.C.; Tsai, C.H.; Hsu, H.C.; Tang, C.H. Transforming growth factor beta1 enhances heme oxygenase 1 expression in human synovial fibroblasts by inhibiting microRNA 519b synthesis. PLoS ONE 2017, 12, e0176052. [CrossRef] [PubMed]

3. Liu, S.-C.; Tsai, C.-H.; Wu, T.-Y.; Tsai, C.-H.; Tsai, F.-J.; Chung, J.-G.; Huang, C.-Y.; Yang, J.-S.; Hsu, Y.-M.; Yin, M.-C.; et al. Soya-cerebroside reduces IL-1 $\beta$-induced MMP-1 production in chondrocytes and inhibits cartilage degradation: Implications for the treatment of osteoarthritis. Food Agric. Immunol. 2019, 30, 620-632. [CrossRef]

4. Benito, M.J.; Veale, D.J.; FitzGerald, O.; van den Berg, W.B.; Bresnihan, B. Synovial tissue inflammation in early and late osteoarthritis. Ann. Rheum. Dis. 2005, 64, 1263-1267. [CrossRef] [PubMed]

5. Dehghan, M.; Asgharian, S.; Khalesi, E.; Ahmadi, A.; Lorigooini, Z. Comparative study of the effect of Thymus daenensis gel 5\% and diclofenac in patients with knee osteoarthritis. Biomedicine (Taipei) 2019, 9, 9. [CrossRef] [PubMed]

6. Sellam, J.; Berenbaum, F. The role of synovitis in pathophysiology and clinical symptoms of osteoarthritis. Nat. Rev. Rheumatol. 2010, 6, 625-635. [CrossRef]

7. Xu, Z.Y.; Liu, Y.L.; Lin, J.B.; Cheng, K.L.; Wang, Y.G.; Yao, H.L.; Wei, P.; Wu, H.Y.; Su, W.W.; Shaw, P.C.; et al. Preparative expression and purification of a nacreous protein N16 and testing its effect on osteoporosis rat model. Int. J. Biol. Macromol. 2018, 111, 440-445. [CrossRef]

8. MacDonald, I.J.; Liu, S.C.; Su, C.M.; Wang, Y.H.; Tsai, C.H.; Tang, C.H. Implications of angiogenesis involvement in arthritis. Int. J. Mol. Sci. 2018, 19, 2012. [CrossRef]

9. Patel, J.; Donovan, P.; Khosrotehrani, K. Concise review: Functional definition of endothelial progenitor cells: A molecular perspective. Stem Cells Transl. Med. 2016, 5, 1302-1306. [CrossRef]

10. Kiewisz, J.; Kaczmarek, M.M.; Pawlowska, A.; Kmiec, Z.; Stompor, T. Endothelial progenitor cells participation in cardiovascular and kidney diseases: A systematic review. Acta Biochim. Pol. 2016, 63, 475-482. [CrossRef]

11. Asahara, T.; Masuda, H.; Takahashi, T.; Kalka, C.; Pastore, C.; Silver, M.; Kearne, M.; Magner, M.; Isner, J.M. Bone marrow origin of endothelial progenitor cells responsible for postnatal vasculogenesis in physiological and pathological neovascularization. Circ. Res. 1999, 85, 221-228. [CrossRef] [PubMed]

12. Peplow, P.V. Influence of growth factors and cytokines on angiogenic function of endothelial progenitor cells: A review of in vitro human studies. Growth Factors 2014, 32, 83-116. [CrossRef] [PubMed] 
13. Su, C.M.; Hsu, C.J.; Tsai, C.H.; Huang, C.Y.; Wang, S.W.; Tang, C.H. Resistin promotes angiogenesis in endothelial progenitor cells through inhibition of microRNA206: Potential implications for rheumatoid arthritis. Stem Cells 2015, 33, 2243-2255. [CrossRef] [PubMed]

14. Chen, C.Y.; Su, C.M.; Hsu, C.J.; Huang, C.C.; Wang, S.W.; Liu, S.C.; Chen, W.C.; Fuh, L.J.; Tang, C.H. CCN1 promotes VEGF production in osteoblasts and induces endothelial progenitor cell angiogenesis by inhibiting miR-126 expression in rheumatoid arthritis. J. Bone Miner. Res. Off. J. Am. Soc. Bone Miner. Res. 2017, 32, 34-45. [CrossRef] [PubMed]

15. Macdonald, I.J.; Liu, S.C.; Huang, C.C.; Kuo, S.J.; Tsai, C.H.; Tang, C.H. Associations between adipokines in arthritic disease and implications for obesity. Int. J. Mol. Sci. 2019, 20, 1505. [CrossRef]

16. Wu, M.H.; Tsai, C.H.; Huang, Y.L.; Fong, Y.C.; Tang, C.H. Visfatin promotes IL-6 and TNF-alpha production in human synovial fibroblasts by repressing miR-199a-5p through ERK, p38 and JNK signaling pathways. Int. J. Mol. Sci. 2018, 19, 190. [CrossRef]

17. Tong, K.M.; Chen, C.P.; Huang, K.C.; Shieh, D.C.; Cheng, H.C.; Tzeng, C.Y.; Chen, K.H.; Chiu, Y.C.; Tang, C.H. Adiponectin increases MMP-3 expression in human chondrocytes through AdipoR1 signaling pathway. J. Cell. Biochem. 2011, 112, 1431-1440. [CrossRef]

18. Tilg, H.; Moschen, A.R. Adipocytokines: Mediators linking adipose tissue, inflammation and immunity. Nat. Rev. Immunol. 2006, 6, 772-783. [CrossRef]

19. Liao, L.; Chen, Y.; Wang, W. The current progress in understanding the molecular functions and mechanisms of visfatin in osteoarthritis. J. Bone Miner. Metab. 2016, 34, 485-490. [CrossRef]

20. Fioravanti, A.; Giannitti, C.; Cheleschi, S.; Simpatico, A.; Pascarelli, N.A.; Galeazzi, M. Circulating levels of adiponectin, resistin, and visfatin after mud-bath therapy in patients with bilateral knee osteoarthritis. Int. J. Biometeorol. 2015, 59, 1691-1700. [CrossRef]

21. Kuo, S.J.; Liu, S.C.; Huang, Y.L.; Tsai, C.H.; Fong, Y.C.; Hsu, H.C.; Tang, C.H. TGF-beta1 enhances FOXO3 expression in human synovial fibroblasts by inhibiting miR-92a through AMPK and p38 pathways. Aging 2019, 11, 4075-4089. [CrossRef] [PubMed]

22. Wu, T.J.; Lin, C.Y.; Tsai, C.H.; Huang, Y.L.; Tang, C.H. Glucose suppresses IL-1beta-induced MMP-1 expression through the FAK, MEK, ERK, and AP-1 signaling pathways. Environ. Toxicol. 2018, 33, 1061-1068. [CrossRef] [PubMed]

23. Wang, S.W.; Liu, S.C.; Sun, H.L.; Huang, T.Y.; Chan, C.H.; Yang, C.Y.; Yeh, H.I.; Huang, Y.L.; Chou, W.Y.; Lin, Y.M.; et al. CCL5/CCR5 axis induces vascular endothelial growth factor-mediated tumor angiogenesis in human osteosarcoma microenvironment. Carcinogenesis 2015, 36, 104-114. [CrossRef] [PubMed]

24. Wu, M.H.; Huang, C.Y.; Lin, J.A.; Wang, S.W.; Peng, C.Y.; Cheng, H.C.; Tang, C.H. Endothelin-1 promotes vascular endothelial growth factor-dependent angiogenesis in human chondrosarcoma cells. Oncogene 2014, 33, 1725-1735. [CrossRef] [PubMed]

25. Wang, M.; Chao, C.C.; Chen, P.C.; Liu, P.I.; Yang, Y.C.; Su, C.M.; Huang, W.C.; Tang, C.H. Thrombospondin enhances RANKL-dependent osteoclastogenesis and facilitates lung cancer bone metastasis. Biochem. Pharmacol. 2019, 166, 23-32. [CrossRef]

26. Liu, J.F.; Lee, C.W.; Tsai, M.H.; Tang, C.H.; Chen, P.C.; Lin, L.W.; Lin, C.Y.; Lu, C.H.; Lin, Y.F.; Yang, S.H.; et al. Thrombospondin 2 promotes tumor metastasis by inducing matrix metalloproteinase-13 production in lung cancer cells. Biochem. Pharmacol. 2018, 155, 537-546. [CrossRef]

27. Lee, H.P.; Chen, P.C.; Wang, S.W.; Fong, Y.C.; Tsai, C.H.; Tsai, F.J.; Chung, J.G.; Huang, C.Y.; Yang, J.S.; Hsu, Y.M.; et al. Plumbagin suppresses endothelial progenitor cell-related angiogenesis in vitro and in vivo. J. Funct. Foods 2019, 52, 537-544. [CrossRef]

28. Lee, H.P.; Wang, S.W.; Wu, Y.C.; Lin, L.W.; Tsai, F.J.; Yang, J.S.; Li, T.M.; Tang, C.H. Soya-cerebroside inhibits VEGF-facilitated angiogenesis in endothelial progenitor cells. Food Agric. Immunol. 2020, 31, 193-204. [CrossRef]

29. Lee, H.P.; Wang, S.W.; Wu, Y.C.; Tsai, C.H.; Tsai, F.J.; Chung, J.G.; Huang, C.Y.; Yang, J.S.; Hsu, Y.M.; Yin, M.C.; et al. Glucocerebroside reduces endothelial progenitor cell-induced angiogenesis. Food Agric. Immunol. 2019, 30, 1033-1045. [CrossRef]

30. Su, C.M.; Tang, C.H.; Chi, M.J.; Lin, C.Y.; Fong, Y.C.; Liu, Y.C.; Chen, W.C.; Wang, S.W. Resistin facilitates VEGF-C-associated lymphangiogenesis by inhibiting miR-186 in human chondrosarcoma cells. Biochem. Pharmacol. 2018, 154, 234-242. [CrossRef] 
31. Wu, K.M.; Hsu, Y.M.; Ying, M.C.; Tsai, F.J.; Tsai, C.H.; Chung, J.G.; Yang, J.S.; Tang, C.H.; Cheng, L.Y.; Su, P.H.; et al. High-density lipoprotein ameliorates palmitic acid-induced lipotoxicity and oxidative dysfunction in $\mathrm{H} 9 \mathrm{c} 2$ cardiomyoblast cells via ROS suppression. Nutr. Metab. 2019, 16, 36. [CrossRef] [PubMed]

32. Yang, Y.C.; Chiou, P.C.; Chen, P.C.; Liu, P.Y.; Huang, W.C.; Chao, C.C.; Tang, C.H. Melatonin reduces lung cancer stemness through inhibiting of PLC, ERK, p38, beta-catenin, and Twist pathways. Environ. Toxicol. 2019, 34, 203-209. [CrossRef] [PubMed]

33. Liu, S.C.; Chuang, S.M.; Hsu, C.J.; Tsai, C.H.; Wang, S.W.; Tang, C.H. CTGF increases vascular endothelial growth factor-dependent angiogenesis in human synovial fibroblasts by increasing miR-210 expression. Cell Death Dis. 2014, 5, e1485. [CrossRef] [PubMed]

34. Wang, C.Q.; Huang, Y.W.; Wang, S.W.; Huang, Y.L.; Tsai, C.H.; Zhao, Y.M.; Huang, B.F.; Xu, G.H.; Fong, Y.C.; Tang, C.H.; et al. Amphiregulin enhances VEGF-A production in human chondrosarcoma cells and promotes angiogenesis by inhibiting miR-206 via FAK/c-Src/PKCdelta pathway. Cancer Lett. 2017, 385, 261-270. [CrossRef] [PubMed]

35. Su, C.M.; Chiang, Y.C.; Huang, C.Y.; Hsu, C.J.; Fong, Y.C.; Tang, C.H. Osteopontin promotes oncostatin M production in human osteoblasts: Implication of rheumatoid arthritis therapy. J. Immunol. 2015, 195, 3355-3364. [CrossRef]

36. Wang, C.J.; Cheng, J.H.; Chou, W.Y.; Hsu, S.L.; Chen, J.H.; Huang, C.Y. Changes of articular cartilage and subchondral bone after extracorporeal shockwave therapy in osteoarthritis of the knee. Int. J. Med. Sci. 2017, 14, 213-223. [CrossRef]

37. Ammendola, M.; Leporini, C.; Luposella, M.; Sacco, R.; Sammarco, G.; Russo, E.; Patruno, R.; De Sarro, G.; Ranieri, G. Targeting endothelial progenitor cells in cancer as a novel biomarker and anti-angiogenic therapy. Curr. Stem Cell Res. Ther. 2015, 10, 181-187. [CrossRef]

38. Li, T.M.; Liu, S.C.; Huang, Y.H.; Huang, C.C.; Hsu, C.J.; Tsai, C.H.; Wang, S.W.; Tang, C.H. YKL-40-induced inhibition of miR-590-3p promotes interleukin-18 expression and angiogenesis of endothelial progenitor cells. Int. J. Mol. Sci. 2017, 18, 920. [CrossRef]

39. Chen, S.S.; Tang, C.H.; Chie, M.J.; Tsai, C.H.; Fong, Y.C.; Lu, Y.C.; Chen, W.C.; Lai, C.T.; Wei, C.Y.; Tai, H.C.; et al. Resistin facilitates VEGF-A-dependent angiogenesis by inhibiting miR-16-5p in human chondrosarcoma cells. Cell Death Dis. 2019, 10, 31. [CrossRef]

40. Su, C.M.; Wang, I.C.; Liu, S.C.; Sun, Y.; Jin, L.; Wang, S.W.; Lee, H.P.; Tseng, W.P.; Tang, C.H. Hypoxia induced mitogenic factor (HIMF) triggers angiogenesis by increasing interleukin-18 production in myoblasts. Sci. Rep. 2017, 7, 7393. [CrossRef]

41. Taipaleenmaki, H. Regulation of bone metabolism by microRNAs. Curr. Osteoporos. Rep. 2018, 16, 1-12. [CrossRef] [PubMed]

42. Gao, B.; Gao, W.; Wu, Z.; Zhou, T.; Qiu, X.; Wang, X.; Lian, C.; Peng, Y.; Liang, A.; Qiu, J.; et al. Melatonin rescued interleukin 1beta-impaired chondrogenesis of human mesenchymal stem cells. Stem Cell Res. Ther. 2018, 9, 162. [CrossRef]

43. Mathiessen, A.; Conaghan, P.G. Synovitis in osteoarthritis: Current understanding with therapeutic implications. Arthritis Res. Ther. 2017, 19, 18. [CrossRef] [PubMed]

44. Hamilton, J.L.; Nagao, M.; Levine, B.R.; Chen, D.; Olsen, B.R.; Im, H.J. Targeting VEGF and its receptors for the treatment of osteoarthritis and associated pain. J. Bone Miner. Res. Off. J. Am. Soc. Bone Miner. Res. 2016, 31, 911-924. [CrossRef] [PubMed]

45. Chen, P.C.; Liu, J.F.; Fong, Y.C.; Huang, Y.L.; Chao, C.C.; Tang, C.H. CCN3 facilitates Runx2 and osterix expression by inhibiting miR-608 through PI3K/Akt signaling in osteoblasts. Int. J. Mol. Sci. 2019, 20, 3300. [CrossRef]

46. Tzeng, H.E.; Tang, C.H.; Wu, S.H.; Chen, H.T.; Fong, Y.C.; Lu, Y.C.; Chen, W.C.; Huang, H.D.; Lin, C.Y.; Wang, S.W.; et al. CCN6-mediated MMP-9 activation enhances metastatic potential of human chondrosarcoma. Cell Death Dis. 2018, 9, 955. [CrossRef]

47. Karar, J.; Maity, A. PI3K/AKT/mTOR pathway in angiogenesis. Front. Mol. Neurosci. 2011, 4, 51. [CrossRef]

48. Samakova, A.; Gazova, A.; Sabova, N.; Valaskova, S.; Jurikova, M.; Kyselovic, J. The PI3k/Akt pathway is associated with angiogenesis, oxidative stress and survival of mesenchymal stem cells in pathophysiologic condition in ischemia. Physiol. Res. 2019, 68, S131-S138. [CrossRef] 
49. Nugent, M. MicroRNAs: Exploring new horizons in osteoarthritis. Osteoarthr. Cartil. 2016, 24, 573-580. [CrossRef]

50. Al-Modawi, R.N.; Brinchmann, J.E.; Karlsen, T.A. Multi-pathway protective effects of MicroRNAs on human chondrocytes in an in vitro model of osteoarthritis. Mol. Ther. Nucleic Acids 2019, 17, 776-790. [CrossRef]

(C) 2020 by the authors. Licensee MDPI, Basel, Switzerland. This article is an open access article distributed under the terms and conditions of the Creative Commons Attribution (CC BY) license (http://creativecommons.org/licenses/by/4.0/). 